Pacific Journal of Mathematics

MULTIPLICATION ALTERATION AND RELATED RIGIDITY 


\section{MULTIPLICATION ALTERATION AND RELATED RIGIDITY PROPERTIES OF ALGEBRAS}

\section{DAve RiffelmacheR}

Given an algebra $C$ over a commutative ring $k$ and an element (called a $C$-two-cocycle) $\sigma=\sum_{i} a_{i} \otimes b_{i} \otimes c_{i}$ in $C \otimes \otimes_{k}$ $C \otimes_{k} C$ satisfying certain relations, Sweedler defined a new multiplication $*$ on $C$ by $x * y=\sum_{i} a_{i} x b_{i} y c_{i}$ for all $x, y$ in $C$ and denoted $C$ with this new multiplication by $C^{\sigma}$. This paper studies three rigidity properties which arise by asking whether:

(i) $C^{\sigma} \simeq C$ as algebras;

(ii) a certain functor from the category of $C$-bimodules to the category of $C^{\sigma}$-bimodules is an equivalence;

(iii) a certain functor from the category of algebras over $C$ to the category of algebras over $C^{\sigma}$ is an equivalence. For certain algebras over a field $k$ (including finite dimensional algebras possessing a Wedderburn factor), these rigidity properties are shown to be equivalent to (respectively): (i) all $k$-separable subalgebras $B$ of $C$ are commutative and for a separability idempotent $\sum_{i} x_{i} \otimes y_{i}$ of $B,\left\{c \in C \mid \sum_{i} x_{i} c y_{i}=0\right\}$ is an ideal with square $\{0\}$; (ii) all $k$-separable subalgebras of $C$ are central; (iii) $k$ is the only $k$-separable subalgebra of $C$.

We recall Sweedler's basic definitions [7] and determine some elementary properties of multiplication alteration in $\S \S 1$ and 2. The behavior of an algebra under alteration by Waterhouse's $C$-twococycle $\sigma_{e}=e \otimes 1+1 \otimes e-(e \otimes 1)(1 \otimes e)$ associated with a $k$-separable subalgebra $B$ of $C$ having separability idempotent $e$ is studied in $\S 3$.

Section 4 introduces the notion of dominance: the $k$-algebra $C$ is said to dominate the $k$-algebra $D$ (written $C>D$ ) if there is a $C$-two-cocycle $\sigma$ with $D \simeq C^{\sigma} . \quad C$ is called rigid if $C>D$ implies $D \simeq C$. Dominance is a partial order on the class of $k$-algebras. In the course of proving this an alternate characterization of a $C$-twococycle $\sigma$ in terms of the existence of a certain functor $F^{\sigma}: A(C) \rightarrow A\left(C^{o}\right)$ is given. (For any $k$-algebra $D, A(D)$ is the category of $k$-algebras over $D$.) We provide a dominance description of the central simple algebras over a field $k$ as the "highly nonrigid" algebras and characterize those algebras over a perfect field $k$ with nilpotent Jacobson radical $J(C)$ and $k$-dim $C / J(C)$ finite which are rigid. The main step in our study of rigidity is a theorem which states that if the kernel of an idempotent algebra endomorphism $p$ of $C$ satisfies a certain nilpotency condition every $C$-two-cocycle $\sigma$ is "equivalent" to the $p(C)$-two cocycle $p(\sigma)$ (cf. Theorem 4.7). 
Section 5 deals with a notion of rigidity on the bimodule level. If $\sigma=\sum_{i} a_{i} \otimes b_{i} \otimes c_{i}$ is a $C$-two-cocycle and $M$ is an object of the category $M(C)$ of $C$-bimodules, we define actions of $C^{\sigma}$ on $M$ by $x^{\sigma} * m=\sum_{i} a_{i} x b_{i} m c_{i}$ and $m * x^{\sigma}=\sum_{i} a_{i} m b_{i} x c_{i}$ for all $x$ in $C, m$ in $M$. Denoting the resulting $C^{\sigma}$-bimodule by $M^{o}$, we obtain a functor ()$^{\sigma}: M(C) \rightarrow M\left(C^{o}\right)$ taking $M$ to $M^{o}$ which we show can also be described as the change of rings functor associated with a certain algebra map $\varphi_{\sigma}: C^{\sigma} \otimes_{k} C^{0} \rightarrow C \otimes_{k} C^{0} . \quad C$ is called modularly rigid (modularly semi-rigid) if ( $)^{\sigma}$ is an equivalence (dense) for all $C$-two-cocycles $\sigma$. If $k$ is a field, we find ( $)^{\sigma_{e}}$ dense for some separability idempotent $e$ of $B \subseteq C$ implies $B$ is central in $C$. We use this to prove: If $k$ is a field, and $C$ is a $k$-algebra with nilpotent Jacobson radical $J(C)$ and $C / J(C)$ locally finite, then $C$ is modularly rigid iff $C$ is modularly semi-rigid iff all $k$-separable subalgebras of $C$ are central.

As mentioned above, $\sigma$ being a $C$-two-cocycle is equivalent to the existence of a certain functor $F^{o}: A(C) \rightarrow A\left(C^{o}\right)$. In $\S 6$ we study these functors. We show that if $C$ is commutative and $\sigma$ is an Amitsur (i.e., invertible) $C$-two-cocycle, then $F^{\sigma}$ is an equivalence of categories. $C$ is called categorically rigid (categorically semi-rigid) if $F^{\sigma}$ is an equivalence (dense) for all $C$-two-cocycles $\sigma$. The paper concludes with a theorem relating categorically rigid algebras and algebras with all two-cocycles invertible. This theorem includes: If $k$ is a field, a $k$-algebra $C$ with nilpotent Jacobson radical $J(C)$ and $C / J(C)$ locally finite is categorically rigid iff $C$ is categorically semi-rigid iff $C$ has no nontrivial $k$-separable subalgebras iff all $C$ two-cocycles are invertible.

The author wishes to thank Moss Sweedler for suggesting the study of these rigidity notions as a thesis problem and for directing this research.

1. Review of basic notions. Throughout this paper $k$ will always denote at least a commutative ring with unit 1 . By an algebra over $k$ or a $k$-algebra we mean an associative, unitary algebra over $k$. Unadorned $\otimes$, Hom represent $\otimes_{k}, \mathrm{Hom}_{k}$ respectively. For any $k$-algebra $C$, we denote the $n$-fold tensor product $C \otimes \cdots \otimes C$ by $C^{\otimes n}$. Given a map $C \stackrel{f}{\rightarrow} D$ of $k$-algebras, we have an induced algebra map $C^{\otimes^{n}} \rightarrow D^{\otimes n}$ for each $n$ given by $x_{1} \otimes \cdots \otimes x_{n} \mapsto f\left(x_{1}\right) \otimes \cdots \otimes f\left(x_{n}\right)$ for $x_{i}$ in $C$ which we denote by $f^{\otimes n}$ or by $f$ if no confusion seems likely. If $C$ is a $k$-algebra, we denote its opposite $k$-algebra by $C^{0}$ and we call a left $C \otimes C^{0}$-module a $C$-bimodule. By an ideal of the $k$-algebra $C$ we mean a two-sided ideal of $C$. $J(C)$ denotes the Jacobson radical of $C$ and $Z(C)$ denotes the center of $C$. By a central simple algebra over the field $k$ we mean a finite $k$-dimensional 
$k$-algebra $C$ with no proper ideals and $Z(C)=k$. Semi-simple means that the Jacobson radical is trivial and the descending chain condition on left ideals holds.

In this section we give a brief review of the theory of multiplication alteration by two-cocycles introduced by Sweedler [7]. Given an algebra $C$ over the commutative ring $k$, let $\sigma=\sum_{i} a_{i} \otimes b_{i} \otimes c_{i}$ be in $C \otimes C \otimes C$. We form a new $k$-algebra $C^{\sigma}$ as follows. As an abelian group, $C^{\sigma}$ is equal to $C$. For any $x$ in $C$ we use the notation $x^{\sigma}$ to indicate that we are considering $x$ as an element of $C^{\sigma}$. We define the product $*$ of any two elements $x^{\sigma}$ and $y^{\sigma}$ in $C^{\sigma}$ by

$$
x^{\sigma} * y^{\sigma}=\left(\sum_{i} a_{\imath} x b_{i} y c_{i}\right)^{\sigma} .
$$

Definition 1.1. $\sigma$ is called a $C$-two-cocycle if

$$
\sum_{i, j} a_{i} a_{j} \otimes b_{j} \otimes c_{j} b_{i} \otimes c_{i}=\sum_{i, j} a_{i} \otimes b_{i} a_{j} \otimes b_{j} \otimes c_{j} c_{i}
$$

and there is an element $e_{o}$ in $C$ with

$$
\sum_{i} \alpha_{i} e_{\sigma} b_{\imath} \otimes c_{i}=1 \otimes 1=\sum_{i} \alpha_{\imath} \otimes b_{i} e_{\sigma} c_{i} .
$$

If $\sigma$ is a $C$-two-cocycle $C^{\sigma}$ is an associative $k$-algebra with unit element $e_{\sigma}^{\sigma}$. This paper may be briefly described as follows: Given a $k$-algebra $C$ and an arbitrary $C$-two-cocycle $\sigma$, we "compare" $C^{\sigma}$ with $C$. In $\S \S 4$ through 6 we investigate three ways of "comparing" $C^{o}$ with $C$, including whether $C^{o} \simeq C$ as $k$-algebras.

EXAMPLE 1.2. Let $C$ be a commutative $k$-algebra and $\sigma=$ $\sum_{i} a_{i} \otimes b_{i} \otimes c_{i}$ be a $C$-two-cocycle. From (1.1b) $\left(\sum_{i} a_{i} b_{i} c_{i}\right) e_{\sigma}=1$ and hence $e_{\sigma}$ is invertible in $C$ with $e_{\sigma}^{-1}=\sum_{i} a_{i} b_{i} c_{i}$. Since $x^{\sigma} * y^{\sigma}=\left(x y e_{\sigma}^{-1}\right)^{\sigma}$ for any $x, y$ in $C$, the $k$-linear map $C \rightarrow C^{o}$ given by $x \mapsto\left(x e_{0}\right)^{\sigma}$ is a $k$-algebra map and is bijective since $e_{\sigma}$ is invertible. Thus $C^{\sigma} \simeq C$.

Let $\sigma=\sum_{i} a_{i} \otimes b_{i} \otimes c_{i}$ and $\tau=\sum_{i} r_{i} \otimes s_{i} \otimes t_{i}$ be $C$-two-cocycles. Associated with any element $\delta=\sum_{i} u_{i} \otimes v_{i}$ in $C \otimes C$ we have a linear map $R^{\delta}: C^{\sigma} \rightarrow C^{\tau}$ given by $x^{\sigma} \mapsto\left(\sum_{i} u_{i} x v_{i}\right)^{\tau}$. if

DEFINITION 1.3. $\sigma$ is cohomologous to $\tau$ via $\delta$, denoted $\sigma \sim^{\delta} \tau$,

$$
\begin{gathered}
\sum_{i, j} u_{i} \alpha_{j} \otimes b_{j} \otimes c_{j} v_{i}= \\
\sum_{i, j, l} r_{i} u_{j} \otimes v_{j} s_{i} u_{l} \otimes v_{l} t_{i}, \\
\sum_{i} u_{i} e_{\sigma} v_{i}=e_{\tau} .
\end{gathered}
$$

Thus if $\sigma \sim^{\delta} \tau, R^{\delta}: C^{\sigma} \rightarrow C^{\tau}$ is a $k$-algebra map.

Definition 1.4. $\delta=\sum_{i} u_{i} \otimes v_{i}$ is called vertible if there is an 
element $\bar{\delta}=\sum_{i} \bar{u}_{i} \otimes \bar{v}_{i}$ in $C \otimes C$ with

$$
\sum_{i, j} u_{i} \bar{u}_{j} \otimes \bar{v}_{j} v_{i}=1 \otimes 1=\sum_{i, j} \bar{u}_{i} u_{j} \otimes v_{j} \bar{v}_{i} .
$$

$\bar{\delta}$ is called the verse of $\delta$.

Hence if $\sigma \sim^{\delta} \tau$ with $\delta$ vertible the map $C^{\delta} \stackrel{R^{\delta}}{\rightarrow} C^{\tau}$ is an isomorphism of $k$-algebras with inverse $R^{\bar{\delta}}$. Because of the existence of this nice isomorphism, we say that $\sigma$ and $\tau$ are equivalent if $\sigma \sim^{\delta} \tau$ with $\delta$ vertible.

ExAMPLES 1.5 .

(a) Let $C=k \oplus k$ and $f=(1,0)$. Then

$$
\sigma_{f}=1 \otimes 1 \otimes 1+f \otimes f \otimes 1+1 \otimes f \otimes f-f \otimes 1 \otimes f-1 \otimes f \otimes 1
$$

is a $C$-two-cocycle with $e_{\sigma_{f}}=1$.

(b) Let $C=k[x]$ with $x^{2}=0$. Then

$$
\sigma_{x}=1 \otimes 1 \otimes 1+x \otimes x \otimes 1+1 \otimes x \otimes x-x \otimes 1 \otimes x
$$

is a $C$-two-cocycle with $e_{\sigma_{x}}=1$. In addition, $\sigma_{x} \sim^{\delta} 1 \otimes 1 \otimes 1$ with $\delta=1 \otimes 1-x \otimes x$ vertible.

2. Structure of $C$ inherited by $C^{\sigma}$. Let $\sigma$ be a $C$-two-cocycle. If $I$ is an ideal of $C$, we have an injective map \{ideals of $C\} \rightarrow$ ideals of $\left.C^{\sigma}\right\}$ given by $I \mapsto I^{\sigma}$. Also, $\left(I^{\sigma}\right)^{2}=I^{\sigma} * I^{\sigma} \subseteq(I \cdot I)^{\sigma}=\left(I^{2}\right)^{\sigma}$ and by induction $\left(I^{o}\right)^{n} \subseteq\left(I^{n}\right)^{\sigma}$ for all $n$. Hence, if $J(C)$ is nilpotent $J(C)^{\sigma} \cong J\left(C^{\sigma}\right)$. If $C \stackrel{f}{\rightarrow} D$ is an algebra map, $f^{\otimes 3}(\sigma)$ is a $D$-two-cocycle with $e_{f(\sigma)}=f\left(e_{\sigma}\right)$. In particular, if $I$ is any ideal of $C$ we may take $D=C / I$ and $f$ the canonical projection $C \rightarrow C / I$. If $C \cong D$ we may take $f$ to be the inclusion and in this way view a $C$-two-cocycle as a $D$-two-cocycle. If $C / J(C)$ is commutative and $C \stackrel{\pi}{\rightarrow} C / J(C)$ is the canonical projection, $\{C / J(C)\}^{\pi(\sigma)} \simeq C^{\sigma} / J(C)^{\sigma}$, and $C / J(C)$ and $C^{\sigma} / J(C)^{\sigma}$ are isomorphic by Example 1.2. Thus $J\left\{C^{\sigma} / J(C)^{\sigma}\right\}=\{0\}^{\sigma}$ and $J(C)^{\sigma} \supseteqq J\left(C^{\sigma}\right)$.

For any $x$ in $Z(C)$ and $y$ in $C,(1.1 b)$ implies $\left(x e_{\sigma}\right)^{\sigma} * y^{\sigma}=y^{\sigma} *\left(x e_{\sigma}\right)^{\sigma}$. Therefore $\left(Z(C) e_{\sigma}\right)^{\sigma} \subseteq Z\left(C^{\sigma}\right)$. The map $Z(C) \stackrel{i}{\rightarrow} Z\left(C^{\sigma}\right)$ given by $x \mapsto\left(x e_{\sigma}\right)^{\sigma}$ is an injective algebra map by (1.1b). Suppose $C / J(C)$ is commutative and let $\sigma=\sum_{i} \alpha_{i} \otimes b_{i} \otimes c_{i}$ be a $C$-two-cocycle. Then

$$
\left\{e_{\sigma}+J(C)\right\}\left\{\sum_{i} a_{i} b_{i} c_{i}+J(C)\right\}=1+J(C)
$$

by (1.1b). Therefore $e_{\sigma}+J(C)$ is invertible in $C / J(C)$ which implies that $e_{\sigma}$ is invertible in $C$. If we let $\tau=\sum_{i} \alpha_{i} \otimes e_{\sigma} b_{i} \otimes e_{\sigma} c_{i} e_{\sigma}^{-1}, \tau$ is a $C$-two-cocycle with $e_{\tau}=1$ and $\tau \sim^{\delta} \sigma$, where $\delta=1 \otimes e_{\sigma}$ is vertible.

For convenience, we assemble our preceding comments and two 
easy consequences in the following lemma.

Lemma 2.1. Let $\sigma$ be a C-two-cocycle.

(i) If $I$ is an ideal of $C, I^{\sigma}$ is an ideal of $C^{o}$.

(ii) If $J(C)$ is nilpotent, $J(C)^{\sigma} \cong J\left(C^{\sigma}\right)$.

(iii) If $C / J(C)$ is commutative, $J\left(C^{\sigma}\right) \subseteq J(C)^{\sigma}$.

(iv) There is a k-algebra injection $Z(C) \hookrightarrow Z\left(C^{o}\right)$.

(v) If $C^{\sigma}$ is simple (i.e., has no proper ideals), $C$ is simple.

(vi) If $C^{o}$ has center $k, C$ has center $k$.

3. Waterhouse two-cocycles. In this section, $C$ is a fixed $k$ algebra and $B$ is a $k$-separable subalgebra of $C$. We investigate some properties of a $B$-two-cocycle discovered by Waterhouse. Recall that the $k$-algebra $B$ is separable over $k$ iff there is an element $e=\sum_{i} a_{i} \otimes b_{i}$ in $B \otimes B$ (called a separability idempotent for $B$ over k) with

$$
\begin{aligned}
\sum_{i} a_{i} b_{i} & =1 \\
\sum_{i} x a_{i} \otimes b_{i} & =\sum_{i} a_{i} \otimes b_{i} x \quad \text { for all } x \text { in } B .
\end{aligned}
$$

The reader may verify that $\sigma_{e}=e \otimes 1+1 \otimes e-(e \otimes 1)(1 \otimes e)$ is a $B$-two-cocycle with $e_{o_{e}}=1$.

DEFINITION 3.2. $\sigma_{e}$ is called the Waterhouse two-cocycle associated to the separable $k$-algebra $B$ with separability idempotent $e$.

The Waterhouse two-cocycle $\sigma_{e}$ figures prominently in our work. In fact, Example 1.5(a) is the Waterhouse two-cocycle for $B=k \oplus k$ and separability idempotent $f \otimes f+(1-f) \otimes(1-f), \quad f=(1,0)$. Using (3.1) it can be shown that $\sigma_{e}^{2}=\sigma_{e}$ in $B^{\otimes 3}$. Since $B$ is a subalgebra of $C$, we may view $\sigma_{e}$ as a $C$-two-cocycle as mentioned in $\S 2$. We examine the algebra $C^{\sigma_{e}}$ in detail.

Define $\Gamma_{e}: C \rightarrow C$ by $\Gamma_{e}(x)=\sum_{i} a_{i} x b_{i}$ for any $x$ in $C . \Gamma_{e}$ is a $Z_{c}(B)$-module endomorphism of $C$, where $Z_{c}(B)=\{x$ in $c \mid x b=b x$ for all $b$ in $B$. We have a $Z_{c}(B)$-module decomposition $C=Z_{c}(B) \oplus \operatorname{Ker} \Gamma_{e}$. Let $a, b$ be in $Z_{c}(B), x, y$ be in $\operatorname{Ker} \Gamma_{e}$. Then it is easily seen from the definition of $\sigma_{e}$ that

$$
\begin{aligned}
& a^{\sigma_{e}} * b^{\sigma_{e}}=(a b)^{\sigma_{e}} \\
& a^{\sigma_{e} * y^{\sigma_{e}}}=(a y)^{\sigma_{e}} \\
& x^{\sigma_{e} * b^{\sigma_{e}}}=(x b)^{\sigma_{e}} \\
& x^{\sigma_{e} * y^{\sigma_{e}}}=0^{\sigma_{e}} \text {. }
\end{aligned}
$$

Thus $\left(\operatorname{Ker} \Gamma_{e}\right)^{\sigma_{e}}$ is an ideal of $C^{\sigma_{e}}$ with $\left(\operatorname{Ker} \Gamma_{e}\right)^{\sigma_{e}} *\left(\operatorname{Ker} \Gamma_{e}\right)^{\sigma_{e}}=\{0\}^{\sigma_{e}}$.

EXAMPLe 3.4. Let $C$ be a central simple algebra of dimension 
$n$ over a field $k$ and choose a separability idempotent $e$ for $C$ over $k$. If $x_{1}, \cdots, x_{n-1}$ are indeterminates over $k$,

$$
C^{\sigma_{e}} \simeq k\left[x_{1}, \cdots, x_{n-1}\right] /\left\langle\left\{x_{i} x_{j}\right\}_{i, j=1}^{n-1}\right\rangle .
$$

4. Rigidity. Using the method of multiplication alteration by two-cocycles, we introduce a partial order on the class of $k$-algebras and study a related rigidity property.

Definition 4.1 (Sweedler). Let $C$ and $D$ be algebras over the commutative ring $k$. We say that $C$ dominates $D$, written $C>D$, if there is a $C$-two-cocycle $\sigma$ with $D \simeq C^{\sigma} . \quad C$ is called rigid if $C>D$ implies that $D \simeq C$.

Since for any $k$-algebra $C$ the element $1 \otimes 1 \otimes 1$ is a $C$-twococycle, dominance is reflexive. To prove that dominance is transitive we first develop another approach to $C$-two-cocycles. Let $A(C)$ denote the category of $k$-algebras over $C$. The objects of $A(C)$ are $k$-algebra maps $C \stackrel{f}{\rightarrow} D$ with $D$ a $k$-algebra. The morphisms are obvious. Let $\mathscr{A}(C)$ denote the category with objects $C \stackrel{f}{\rightarrow} D$, where $C, D$ are $k$-modules with multiplications (i.e., $k$-linear maps $C \otimes C \rightarrow C$ and $D \otimes D \rightarrow D$ ) and $f$ is a multiplicative $k$-module map. Again take the obvious morphisms. Note that $A(C)$ is a subcategory of $\mathscr{A}(C)$.

Given any element $\sigma=\sum_{i} a_{i} \otimes b_{i} \otimes c_{i}$ in $C \otimes C \otimes C$ and an object $C \stackrel{f}{\rightarrow} D$ of $A(C)$, we have an object $C^{\sigma} \stackrel{f^{\sigma}}{\rightarrow} D^{f(\sigma)}$ of $\mathscr{A}\left(C^{\sigma}\right)$ with the multiplication in $D^{f(\sigma)}$ given by

$$
\left.x^{f(\sigma)} * y^{f(\sigma)}=\sum_{i} f\left(a_{i}\right) x f\left(b_{i}\right) y f\left(c_{i}\right)\right)^{f(\sigma)}
$$

and $f^{\sigma}\left(x^{o}\right)=f(x)^{f(o)}$ for $x, y$ in $C$. In this manner we obtain a functor $A(C) \stackrel{F^{\sigma}}{\rightarrow} \mathscr{A}\left(C^{o}\right)$.

Notation. For any $k$-algebra $C$, we denote the free algebra obtained by adjoining three noncommuting indeterminants $X, Y, Z$ by $C\{X, Y, Z\}$.

The following lemma gives a characterization of a $C$-two-cocycle $\sigma$ in terms of the functor $F^{\sigma}$.

Lemma 4.2 (Sweedler). Let $C$ be an algebra over the commutative ring $k$ and $\sigma$ be in $C \otimes C \otimes C$. The following are equivalent:

(i) $\sigma$ is a C-two-cocycle.

(ii) The image of $F^{\circ}$ lies in $A\left(C^{\sigma}\right)$, i.e., $F^{o}$ is a functor from $A(C)$ to $A\left(C^{o}\right)$.

(iii) $C\{X, Y, Z\}^{\circ}$ is an associative unitary k-algebra. 
Proof. (i) $\Rightarrow$ (ii). If $\sigma$ is a $C$-two-cocycle and $C \stackrel{f}{\rightarrow} D$ is in $A(C)$, $f(\sigma)$ is a $D$-two-cocycle and hence $C^{\sigma} \stackrel{f^{\sigma}}{\rightarrow} D^{f(\sigma)}$ is in $A\left(C^{\sigma}\right)$.

(ii) $\Rightarrow$ (iii). $\quad C \stackrel{f}{\rightarrow} C\{X, Y, Z\}$ where $f(c)=c$ for all $c$ in $C$ is an object of $A(C)$ and thus by hypothesis $C^{o} \stackrel{f^{o}}{\rightarrow} C\{X, Y, Z\}^{f(\sigma)}$ is an object of $A\left(C^{o}\right)$. Hence $C\{X, Y, Z\}^{f(\sigma)}=C\{X, Y, Z\}^{o}$ is an associative unitary $k$-algebra.

(iii) $\Rightarrow$ (i). The unit $e_{\sigma}^{o}$ of $C\{X, Y, Z\}^{o}$ must lie in $C$ and we have $X^{\sigma} * e_{\sigma}^{\sigma}=X^{\sigma}=e_{\sigma}^{\sigma} * X^{\sigma}$ which implies (1.1b). By associativity, $X^{\sigma} *\left(Y^{\sigma} * Z^{\sigma}\right)=$ $\left(X^{\sigma} * Y^{\sigma}\right) * Z^{\sigma}$ which implies (1.1a). Thus $\sigma$ is a $C$-two-cocycle.

Propositron 4.3. Dominance is transitive.

Proof. Suppose we have a $C$-two-cocycle $\sigma=\sum_{i} a_{i} \otimes b_{i} \otimes c_{i}$ and a $C^{\sigma}$-two-cocycle $\tau=\sum_{i} d_{i}^{\sigma} \otimes e_{i}^{\sigma} \otimes f_{i}^{\sigma}$. Let $x, y$ be in $C$. Then writing out $\left(x^{\sigma}\right)^{\tau} *\left(y^{\sigma}\right)^{\tau}$ shows that we will be done if we can prove that

$$
\gamma=\sum_{i_{1}, i_{2}, i_{3}, i_{4}, j} a_{i_{1}} a_{i_{2}} a_{i_{3}} a_{i_{4}} d_{j} b_{i_{4}} \otimes c_{i_{4}} b_{i_{3}} e_{j} c_{i_{3}} b_{i_{2}} \otimes c_{i_{2}} b_{i_{1}} f_{j} c_{i_{1}}
$$

is a $C$-two-cocycle with $e_{r}=e_{\tau}$ since then $C^{r} \simeq\left(C^{\sigma}\right)^{\tau}$ via $x^{\gamma} \mapsto\left(x^{\sigma}\right)^{\tau}$. By Lemma 4.2 we have functors $F^{o}: A(C) \rightarrow A\left(C^{o}\right)$ and $F^{\tau}: A\left(C^{o}\right) \rightarrow$ $A\left(\left(C^{\sigma}\right)^{\tau}\right)$. The composite $F^{\tau} \circ F^{o}$ is just $F^{\gamma}$. Hence $\gamma$ is a $C$-twococycle by Lemma 4.2. It is easily checked that $e_{r}=e_{\tau}$.

Therefore dominance is a partial order on the class of $k$-algebras. In $\S 6$ we study the functors $A(C) \stackrel{F^{\sigma}}{\rightarrow} A\left(C^{\sigma}\right)$ in detail.

REMARKS 4.4. (a) Example 1.2 shows that commutative $k$ algebras are rigid.

(b) If $C=M(n, k)$ and $\sigma=\sigma_{e}$ is a Waterhouse two-cocycle for $C, C^{\sigma}$ is commutative (cf. Example 3.4). Hence dominance is not symmetric.

The following two theorems provide a dominance characterization of central simple $k$-algebras.

THEOREM 4.5. Let $C$ be an algebra over a field k. If $C$ dominates a separable k-algebra, $C$ is separable over $k$.

Proof. There exists a $C$-two-cocycle $\sigma$ with $C^{\sigma} k$-separable. Hence by [6, Theorem 3.1] $k$-dim $C=k-\operatorname{dim} C^{o}$ is finite. It then follows from Lemma 2.1 that $J(C)^{\sigma} \subseteq J\left(C^{\sigma}\right)=\{0\}^{\sigma}$, proving $C$ to be semi-simple. Since $Z(C) \hookrightarrow Z\left(C^{o}\right)$ by Lemma 2.1 and $Z\left(C^{o}\right)$ is a commutative separable $k$-algebra [ 1 , Theorem III.12], $Z(C)$ is $k$-separable. Therefore $C$ is $k$-separable, again using [1, Theorem III.21]. 
THEOREM 4.6. Let $k$ be a field and $C$ a k-algebra with $k$-dim $C=n$. The following are equivalent:

(a) $C$ dominates a central simple k-algebra.

(b) $C$ is a central simple k-algebra.

(c) $C>D$ for all k-algebras $D$ with $k$-dim $D=n$.

(d) $C>k \oplus \cdots \oplus k$ and $C>k[x] /\left(x^{n}\right)$.

(e) $C$ dominates a separable k-algebra and $C$ dominates a purely inseparable k-algebra.

Proof. Recall that an algebra $A$ over the field $k$ is a purely inseparable $k$-algebra [8, Definition 1] if the contraction map $A \otimes$ $A^{0} \rightarrow A$ given by $a \otimes b^{0} \mapsto a b$ provides an $A \otimes A^{0}$ projective cover of $A$. If $k-\operatorname{dim} A<\infty, A$ is purely inseparable over $k$ iff $A / J(A)$ is a purely inseparable (in the usual sense) field extension of $k$ [8, Corollary 13(b)].

(a) $\Leftrightarrow(b)$. This is clear from the reflexive property of dominance and (v) and (vi) of Lemma 2.1.

(b) $\Rightarrow(\mathrm{c})$. Let $D$ be any $k$-algebra of $k$-dimension $n$. We may then identify $C$ and $D$ as $k$-spaces. Since $C$ is central simple, by $[7,1.3 \mathrm{a}$ and 1.6] we have a linear isomorphism $C \otimes C \otimes C \simeq$ Hom $(C \otimes C, C)$ given by

$$
x_{1} \otimes x_{2} \otimes x_{3} \longmapsto\left(y_{1} \otimes y_{2} \longmapsto x_{1} y_{1} x_{2} y_{2} x_{3}\right) \text {. }
$$

Since a multiplication on $D$ is a linear map $C \otimes C \rightarrow C$ we have an element $\sigma$ in $C^{\otimes 3}$ with $C^{\sigma} \simeq D$ as $k$-algebras. By [7, Proposition 1.6] $\sigma$ is a $C$-two-cocycle, and thus $\mathrm{C}>D$.

$(c) \Rightarrow(d)$. Clear.

$(\mathrm{d}) \Rightarrow(\mathrm{e})$. Clear since $k \oplus \cdots \oplus \oplus$ is $k$-separable and $k[x] /\left(x^{n}\right)$ is $k$-purely inseparable.

$(\mathrm{e}) \Rightarrow(\mathrm{b})$. By Theorem 4.5, $C$ is $k$-separable. In particular, $C$ is a finite $k$-dimensional semi-simple $k$-algebra and $Z(C)$ is $k$-separable. We have a $C$-two-cocycle $\tau$ with $C^{\tau}$ purely inseparable over $k$. Since $Z(C) \hookrightarrow C^{\tau}$ (cf. 2.1 (iv)), $Z(C)$ is purely inseparable $k$ [8, Corollary 7 (c)]. Thus $Z(C)$ is both separable and purely inseparable over $k$, which implies $Z(C)=k$ [8, Corollary $7(a)]$. Since $C$ is semisimple and $Z(C)=k$, it follows that $C$ is simple.

We now study the structure of rigid algebras. The crucial theorem is

THEOREM 4.7. Let k be a commutative ring and $C$ be a k-algebra. Suppose there is a k-algebra map $p: C \rightarrow C$ such that $p^{2}=p$ and $(\operatorname{Ker}(p)) \otimes C^{0}+C \otimes(\operatorname{Ker}(p))^{0} \leqq J\left(C \otimes C^{0}\right)$. Then every $C$-two-cocycle is equivalent to a $p(C)$-two-cocycle. 
Proof. Let $\sigma=\sum_{i} a_{i} \otimes b_{i} \otimes c_{i}$ be a $C$-two-cocycle. Since

$$
\begin{aligned}
{[p \otimes} & \left.1^{0}\right]\left(\sum_{i} p\left(a_{i} e_{\sigma}\right) b_{i} \otimes c_{i}^{0}\right)=\sum_{i} p\left(a_{i} e_{\sigma}\right) p\left(b_{i}\right) \otimes c_{i}^{0} \\
& =\left[p \otimes 1^{0}\right]\left(\sum_{i} a_{i} e_{o} b_{i} \otimes c_{i}^{0}\right) \\
& =\left[p \otimes 1^{0}\right]\left(1 \otimes 1^{0}\right)=1 \otimes 1^{0}
\end{aligned}
$$

and $\operatorname{ker}\left(p \otimes 1^{0}\right) \subseteq J\left(C \otimes C^{0}\right)$ by hypothesis, $\sum_{i} p\left(a_{i} e_{o}\right) b_{i} \otimes c_{i}^{0}$ is invertible in $C \otimes C^{0}$. Thus $\delta_{1}=\sum_{i} p\left(a_{i} e_{o}\right) b_{i} \otimes c_{i}$ is vertible. Denote its verse by $\sum_{i} u_{i} \otimes v_{i} \cdot \tau_{1} \sim^{1} \sigma$ defines a $C$-two-cocycle $\tau_{1}$ with $e_{\tau_{1}}=p\left(e_{\sigma}\right)$ and it follows from the associativity relation for $\sigma$ that

$$
\tau_{1}=\sum_{i, j} p\left(a_{i}\right) \otimes b_{i} u_{j} \otimes v_{j} c_{i}
$$

By an obvious analog of the argument used for $\delta_{1}$ above, one may see that $\delta_{2}=\sum_{i, j} p\left(\alpha_{i}\right) \otimes b_{i} u_{j} p\left(e_{o} v_{j} c_{i}\right)$ is vertible. Call its verse $\sum_{i} x_{i} \otimes y_{i}$. Note that $\sum_{i} x_{i} \otimes y_{i}=\sum_{i} p\left(x_{i}\right) \otimes y_{i}$ by uniqueness of verse (uniqueness of inverse in $C \otimes C^{0}$ ).

$\tau_{2} \sim^{\delta_{2}} \tau_{1}$ defines a $C$-two-cocycle $\tau_{2}$ with $e_{\tau_{2}}=e_{\tau_{1}}$ and it follows from the associativity relation for $\tau_{1}$ that

$$
\tau_{2}=\sum_{i, j, l} p\left(a_{i}\right) x_{j} \otimes y_{j} b_{i} u_{l} \otimes p\left(v_{l} c_{i}\right) .
$$

Thus $\tau_{2}$ is in $p(C) \otimes C \otimes p(C)$.

We claim that $\tau_{2}$ in fact lies in $p(C)^{83}$. To see this, apply the map $\left(1 \otimes m_{e} \otimes 1\right) \circ(1 \otimes p \otimes 1 \otimes 1)$ to the associativity relation for $\tau_{2}$, where $m_{e}: C \otimes C \rightarrow C$ is given by $a \otimes b \mapsto a e_{\tau_{2}} b$. Since $p^{2}=p$, this yields that $\tau_{2}$ is in $p(C)^{\otimes 3}$.

TheOREM 4.8. $k$ perfect field. Let $C$ be a k-algebra with $J(C)$ nilpotent and $C / J(C)$ locally finite (i.e., every finite subset of $C / J(C)$ generates a finite dimensional $k$-algebra). If every $k$-separable subalgebra $B$ of $C$ is commutative and $\operatorname{Ker} \Gamma_{\theta}$ is an ideal of square zero for some separability idempotent $e$ of $B$, then $C$ is rigid.

Proof. Let $\sigma=\sum_{i=1}^{n} a_{i} \otimes b_{i} \otimes c_{i}$ be a $C$-two-cocycle and let $D$ be the subalgebra of $C$ generated by $\left\{a_{i}, b_{i}, c_{i}, e_{o}\right\}_{i=1}^{n} \cup J(C)$. Since $J(C)$ is a nilpotent ideal of $D, J(C) \subseteq J(D)$. The locally finiteness of $C / J(C)$ implies that $D / J(C)$ is finite dimensional. Hence the radical $J(D / J(C)=$ $J(D) / J(C)$ of $D / J(C)$ is nilpotent. Since $J(C)$ is nilpotent, it follows that $J(D)$ is nilpotent.

$D / J(D)$ is $k$-separable. Hence by the Wedderburn Principal Theorem $D=B \oplus J(D)$ for some $k$-separable subalgebra $B$ of $C$. (cf. [4, Theorem 72.19]. To remove the finite dimension restriction on $D$, induct on the index of nilpotency of $J(D)$.) By Theorem $4.7 \sigma$ is 
equivalent to a $B$-two-cocycle. Hence we need only show $C^{\sigma} \simeq C$ if $\sigma$ is in $B^{\otimes 3}$. Since $B$ is commutative by hypothesis, we may assume $e_{\sigma}=1$.

Recall that $C=Z_{C}(B) \oplus \operatorname{Ker} \Gamma_{e}$. By hypothesis we may assume $\operatorname{Ker} \Gamma_{e}$ is an ideal of square zero. For $a, b$ in $Z(C), x, y$ in $\operatorname{Ker} \Gamma_{e}$, we have

$$
\begin{aligned}
a^{\sigma} * b^{\sigma} & =(a b)^{\sigma} \\
a^{\sigma} * y^{\sigma} & =(a y)^{\sigma} \\
x^{\sigma} * b^{\sigma} & =(x b)^{\sigma} \\
x^{\sigma} * y^{\sigma} & =0^{\sigma}=(x y)^{\sigma} .
\end{aligned}
$$

Thus $C^{\sigma} \simeq C$ via $c^{\sigma} \Leftrightarrow c$.

We now study dominance and Waterhouse two-cocycles in order to prove a partial converse of Theorem 4.8.

LeMma 4.9. Let $C$ be a k-algebra with $J(C)=\{0\}$. Suppose that $B$ is a k-separable subalgebra of $C$ with separability idempotent $e$. If $C^{\sigma_{e}} \simeq C, B \subseteq Z(C)$.

Proof. $J\left(C^{\sigma_{e}}\right)=\{0\}^{\sigma_{e}}$ so the nilpotent ideal $\left(\operatorname{Ker} \Gamma_{e}\right)^{\sigma_{e}}$ must be the zero ideal. Hence $C=Z_{C}(B)$ and $B$ is central.

LeMma 4.10. Let $C$ be an algebra over the field $k, B$ a k-separable subalgebra with separability idempotent $e$. If $C^{\sigma_{e}} \simeq C, B$ is commutative.

Proof. Consider the canonical projection $C \stackrel{\pi}{\rightarrow} C / J(C) . \quad \bar{B}=\pi(B)$ is a $k$-separable subalgebra of $\bar{C}=C / J(C)$ with separability idempotent $\bar{e}=\pi(e)$. Since $C^{\sigma}{ }^{\sigma} \simeq C$, we have $\bar{C}^{\sigma \bar{e}} \simeq \bar{C}$. Hence by Lemma $4.9 \bar{B}$ is central in $\bar{C}$. Thus for all $x, y$ in $B x y-y x$ is in $B \cap J(C)$. Since $B$ is finite dimensional over $k, B \cap J(C)$ is a nil ideal of $B$. Because $B$ is separable, $J(B)=\{0\}$ and hence $B \cap J(C)=\{0\}$. Therefore $B$ is commutative.

THeOREM 4.11. Let $k$ be a perfect field and $C$ be a k-algebra with $J(C)$ nilpotent and $k$-dimension of $C / J(C)$ finite. If $C$ is rigid every k-separable subalgebra $B$ of $C$ is commutative and $\operatorname{Ker} \Gamma_{e}$ is an ideal of square zero for some separability idempotent e for $B$.

Proof. Every $k$-separable subalgebra of $C$ is commutative by Lemma 4.10. Using the Wedderburn Principal Theorem we have $C=B_{0} \oplus J(C)$ for some $k$-separable subalgebra $B_{0}$ of $C$. For any separability idempotent $e_{0}$ for $B_{0}, C^{\sigma_{e_{0}}} \simeq C$ implies that there is a $k$ - 
separable subalgebra $B_{1}$ of $C$ with separability idempotent $e_{1}$ such that $B_{1} \simeq B_{0}$ and $\operatorname{Ker} \Gamma_{e_{1}}$ is an ideal of square zero. Since any two Wedderburn factors of $C$ are isomorphic by an inner automorphism of $C$ (cf [4, p. 491]) and any $k$-separable subalgebra of $C$ is contained in some Wedderburn factor, we are done.

Combining Theorems 4.8 and 4.11 we have

THEOREM 4.12. Let $k$ be a perfect field and $C$ be a k-algebra with $J(C)$ nilpotent and $k$-dimension of $C / J(C)$ finite. Then $C$ is rigid iff every $k$-separable subalgebra $B$ of $C$ is commutative and $\operatorname{Ker} \Gamma_{e}$ is an ideal of square zero for some separability idempotent e for $B$.

5. Modular rigidity. Given a $k$-algebra $C$, we denote by $M(C)$ the category of $C$-bimodules. Let $\sigma=\sum_{i} a_{i} \otimes b_{i} \otimes c_{i}$ be a $C$-twococycle. If $M$ is $C$-bimodule, we form a $C^{\sigma}$-bimodule from $M$ in the following manner. Starting with an abelian group $M^{\circ}$ isomorphic with $M$ via $m^{\sigma} \Leftrightarrow m$, we define left and right actions of $C^{\sigma}$ on $M^{o}$ by

$$
\begin{gathered}
x^{\sigma} * m^{\sigma}=\left(\sum_{i} a_{i} x b_{i} m c_{i}\right)^{\sigma} \\
m^{\sigma} * x^{\sigma}=\left(\sum_{i} a_{i} m b_{i} x c_{i}\right)^{\sigma} x \text { in } C, m \text { in } M .
\end{gathered}
$$

Using the defining relations (1.1) of a $C$-two-cocycle, it is readily checked that this provides $M^{o}$ with a $C^{\sigma}$-bimodule structure. Given a $C$-bimodule map $M \stackrel{f}{\rightarrow} N$ we let $M^{\sigma} \stackrel{f^{\sigma}}{\rightarrow} N^{\sigma}$ by $f^{\sigma}\left(m^{\sigma}\right)=f(m)^{\sigma}$. These constructions define a faithful functor from $M(C)$ to $M\left(C^{o}\right)$ which we denote by ()$^{\sigma}$.

We define a linear map

$$
\begin{aligned}
& C^{\sigma} \otimes C^{\sigma^{0}} \stackrel{\varphi_{o}}{\longrightarrow} C \otimes C^{0} \\
& x^{\sigma} \otimes y^{\sigma^{0}} \longrightarrow \sum_{i, j} a_{i} a_{j} x b_{j} \otimes\left(c_{j} b_{i} y c_{i}\right)^{0} .
\end{aligned}
$$

LEMMA 5.1. $\varphi_{\sigma}$ is a map of k-algebras.

Proof. Since $\varphi_{\sigma}$ is linear and $\varphi_{\sigma}\left(e_{\sigma}^{\sigma} \otimes e_{\sigma}^{\sigma^{0}}\right)=1 \otimes 1^{0}$, we need only check that $\varphi_{\sigma}$ is multiplicative. This follows from the two-cocycle associativity relation for $\sigma$ :

$$
\begin{gathered}
\varphi_{\sigma}\left\{\left(x^{\sigma} \otimes y^{\sigma^{0}}\right) *\left(x_{1}^{o} \otimes y_{1}^{\sigma^{0}}\right)\right\}=\varphi_{\sigma}\left\{\sum_{i, j}\left(a_{i} x b_{i} x_{1} c_{i}\right)^{\sigma} \otimes\left(a_{j} y_{1} b_{j} y c_{j}\right)^{\sigma^{0}}\right\} \\
=\sum_{i, j, m, n} a_{m} a_{n} a_{i} x b_{i} x_{1} c_{i} b_{n} \otimes\left(c_{n} b_{m} a_{j} y_{1} b_{j} y c_{j} c_{m}\right)^{0} \\
=\sum_{i, j, m, n} a_{m} a_{i} x b_{i} x_{1} c_{i} b_{m} a_{n} \otimes\left(b_{n} a_{j} y_{1} b_{j} y c_{j} c_{n} c_{m}\right)^{0}
\end{gathered}
$$




$$
\begin{aligned}
& =\sum_{i, j, m, n} a_{m} x b_{m} a_{i} x_{1} b_{i} a_{n} \otimes\left(b_{n} a_{j} y_{1} b_{j} y c_{j} c_{n} c_{i} c_{m}\right)^{0} \\
& =\sum_{i, j, m, n} a_{m} x b_{m} a_{i} a_{n} x_{1} b_{n} \otimes\left(c_{n} b_{i} a_{j} y_{1} b_{j} y c_{j} c_{i} c_{m}\right)^{0} \\
& =\sum_{i, j, m, n} a_{m} x b_{m} a_{i} a_{j} a_{n} x_{1} b_{r_{b}} \otimes\left(c_{n} b_{j} y_{1} c_{j} b_{i} y c_{i} c_{m}\right)^{0} \\
& =\left\{\sum_{m, i} a_{m} x b_{m} a_{i} \otimes\left(b_{i} y c_{i} c_{m}\right)^{0}\right\}\left\{\sum_{j, n} a_{j} a_{n} x_{1} b_{n} \otimes\left(c_{n} b_{j} y_{1} c_{j}\right)^{0}\right\} \\
& =\varphi_{\sigma}\left(x^{\sigma} \otimes y^{\sigma^{0}}\right) \cdot \varphi_{\sigma}\left(x_{1}^{\sigma} \otimes y_{1}^{\sigma^{0}}\right) .
\end{aligned}
$$

The change of rings functor induced by $\phi_{\sigma}$ is the functor that we called ()$^{b}$.

Recall that a functor $F$ from a category $\mathscr{A}$ to a category $\mathscr{B}$ is dense if given any object $B$ of $\mathscr{B}$ there is an object $A$ of $\mathscr{A}$ with $F(A)$ isomorphic to $B$ in $\mathscr{B}$.

Remarks 5.2. (a) $C^{\sigma} \otimes C^{\sigma^{0}}$ is a faithful $C^{\sigma} \otimes C^{\sigma^{0}}$-module. Thus if ()$^{\sigma}$ is dense, $\varphi_{\sigma}$ is injective since $\varphi_{\sigma}\left(\sum_{i} x_{i}^{\sigma} \otimes y_{i}^{\sigma^{0}}\right)=0$ implies $\left(\sum_{i} x_{i}^{o} \otimes y_{i}^{0^{0}}\right) * M^{o}=\{0\}^{\sigma}$ for all $M$ in $M(C)$.

(b) If $\phi_{\sigma}$ is an isomorphism, ( $)^{\sigma}$ is an equivalence of categories.

(c) If $C$ is a finite dimensional algebra over a field $k$, parts (a) and (b) imply that ( $)^{\sigma}$ is an equivalence iff $\varphi_{\sigma}$ is an isomorphism since $k$-dim $C \otimes C^{0}=k$-dim $C^{o} \otimes C^{\sigma^{0}}$.

Definition 5.3 (Sweedler). Let $C$ be a $k$-algebra. We say that $C$ is modularly rigid (modularly semi-rigid) if ()$^{\sigma}$ is an equivalence (dense) for all $C$-two-cocycles $\sigma$.

Note that modular rigidity implies modular semi-rigidity. We will later show that for certain types of algebras over a field $k$, e.g., finite dimensional ones, modular rigidity is equivalent to modular semi-rigidity.

EXAMPLes 5.4. (a) Let $C$ be a commutative ring and $\sigma$ be a $C$-two-cocycle. By Example 1.2, $e_{\sigma}$ is invertible. For $x, y$ in $C$, $\varphi_{\sigma}\left(x^{\sigma} \otimes y^{\sigma^{0}}\right)=\left(x \otimes y^{0}\right)\left(e_{\sigma}^{-1} \otimes e_{\sigma}^{-1^{0}}\right)$. Hence $C$ is modularly rigid by (5.2b).

(b) Let $C=U(2, k)$, the algebra of upper triangular two by two matrices over $k$, and take $\sigma_{e}$ to be the Waterhouse two-cocycle associated with $B=k e_{11} \oplus k e_{22}$ and separability idempotent $e=e_{11} \otimes$ $e_{11}+e_{22} \otimes e_{22}$. Since $\varphi_{\sigma_{e}}\left(e_{12}^{\sigma_{e}} \otimes e_{12}^{\sigma_{e} 0}\right)=0$ by direct calculation, ( $)^{\sigma_{e}}$ is not dense by (5.2a). Thus $U(2, k)$ is rigid (by Theorem 4.7) but not modularly rigid.

The remainder of this section is devoted to studying the structure of modularly rigid algebras over a field $k$. 
LEMma 5.5. Let $C$ be an algebra over a commutative ring $k$. Suppose that $\sigma=\sum_{i} a_{i} \otimes b_{i} \otimes c_{i}$ is a C-two-cocycle and let

$$
z_{\sigma}=\sum_{i, j} a_{i} a_{j} \otimes_{Z(C)} b_{j}^{0} \otimes c_{j} b_{i} \otimes_{Z(C)} c_{i}^{0}
$$

in $C \otimes_{z(C)} C^{0} \otimes C \otimes_{z(C)} C^{0}$. Then if $z_{\sigma}$ is invertible, $\varphi_{\sigma}$ is an isomorphism.

Proof. Let $z_{\sigma}^{-1}=\sum_{l} p_{l} \otimes_{z(C)} r_{l}^{0} \otimes s_{l} \otimes_{z(C)} t_{l}^{0}$. Define a map $\Lambda_{\sigma}$ : $C \otimes C^{0} \rightarrow C^{0} \otimes C^{a^{0}}$ by

$$
\Lambda_{\sigma}\left(\sum_{i} x_{i} \otimes y_{i}^{0}\right)=\sum_{i, l}\left(p_{l} x_{i} r_{l}\right)^{\sigma} \otimes\left(s_{l} y_{i} t_{l}\right)^{\sigma^{0}} .
$$

Then $z_{\sigma}^{-1} z_{\sigma}=1$ implies $\Lambda_{o} \varphi_{\sigma}=I_{C^{\sigma} \otimes C^{0}}$ and $z_{\sigma} z_{\sigma}^{-1}=1$ implies $\varphi_{\sigma} \Lambda_{\sigma}=I_{C \otimes C^{0}}$. Hence $\Lambda_{o}=\varphi_{o}^{-1}$.

In preparation for the next theorem, we need the following

LEMMA 5.6. $k$ field. Let $C$ be an algebraic k-algebra with all $k$-separable subalgebras of $C / J(C)$ central. Then every semi-simple subalgebra of $C / J(C)$ is commutative.

This lemma may be proved using Wedderburn-Artin structure theory and the Jacobson-Noether theorem [5, Theorem 3.2.1].

THEOREM 5.7. $k$ field. Let $C$ be a k-algebra with $J(C)$ nilpotent and $C / J(C)$ locally finite. If all k-separable subalgebras of $C$ are central, $\varphi_{\sigma}$ is an isomorphism for all C-two-cocycles $\sigma$.

Proof. As in the proof of Theorem 4.8, let $\sigma=\sum_{i=1}^{n} a_{i} \otimes b_{i} \otimes c_{i}$ be a $C$-two-cocycle and let $D$ be the subalgebra of $C$ generated by $\left\{a_{i}, b_{i}, c_{i}, e_{o}\right\}_{i=1}^{n} \cup J(C)$. If $\bar{B}$ is any $k$-separable subalgebra of $D / J(D)$, we may lift $\bar{B}$ isomorphically to a $k$-separable subalgebra $B \subseteq D \subseteq C$ by the Wedderburn Principal Theorem. By hypothesis, $B \subseteq Z(C)$, and so also $B \subseteq Z(D)$ and $\bar{B} \subseteq Z(D / J(D))$. Therefore $D / J(D)$ is commutative by Lemma 5.6.

Let $\bar{D}=D / J(D)$. Since $\bar{D}$ is commutative and finite dimensional, there exists a unique maximal $k$-separable subalgebra $\bar{S}$ of $\bar{D}$ and $\bar{D}$ is a purely inseparable $\bar{S}$-algebra (to see this, use structure theory to write $\bar{D}$ as a finite product of field extensions of $k$ ). Lift $\bar{S}$ via the Wedderburn Principal Theorem to a $k$-separable subalgebra $S$ in the center of $C$.

Let $\ddot{z}=\sum_{i, j} a_{i} a_{j} \otimes_{S} b_{j}^{0} \otimes c_{j} b_{i} \otimes_{S} c_{i}^{0}$. We claim that $z$ is invertible. Once this is established, we would be able to complete the proof by noting that then the image $z_{\sigma}$ of $z$ in $C \otimes_{z(C)} C^{0} \otimes C \otimes_{z(C)} C^{0}$ is invertible and hence Lemma 5.5 applies.

Thus we need only show that $z$ is invertible. First, we note 
that $z$ is in $D \otimes{ }_{s} D^{0} \otimes D \otimes{ }_{s} D^{0}$. Since $J(D)$ is nilpotent, $z$ is invertible in $\left(D \otimes{ }_{s} D^{0}\right)^{82}$ iff its image $\bar{z}$ under the natural map

$$
D \otimes{ }_{S} D^{0} \otimes D \otimes{ }_{S} D^{0} \longrightarrow \bar{D} \otimes{ }_{\bar{s}} \bar{D} \otimes \bar{D} \otimes{ }_{\bar{s}} \bar{D}
$$

is invertible.

Because $\bar{D}$ is purely inseparable over $\bar{S}$, the kernel of the contraction map $\bar{D} \otimes \bar{s}_{\bar{D}} \bar{D}^{\circ} \stackrel{m}{\rightarrow} \bar{D}$ is contained in $J\left(\bar{D} \otimes{ }_{\bar{s}} \bar{D}^{0}\right) . \quad J\left(\bar{D} \otimes \bar{s}_{\bar{s}} \bar{D}^{0}\right)$ is nilpotent since $\bar{D} \otimes_{\bar{S}} \bar{D}^{0}$ is a finite dimensional $k$-algebra and hence $\bar{z}$ is invertible iff its image under the map $m \otimes m$ is invertible (note that $m$ is an algebra map since $\bar{D}$ is commutative). Since $\{m \otimes m\}(x)$ clearly has inverse $\bar{e}_{\sigma} \otimes \bar{e}_{o}$, we done.

We use Waterhouse two-cocycles to obtain the converse of the above theorem.

LEMma 5.8. Let $C$ be an algebra over the field $k$ and $B$ a $k$ separable subalgebra of $C$ with separability idempotent $e$. Then if $\varphi_{\sigma_{e}}$ is injective $B$ is central in $C$.

Proof. If $B$ were not central, we would have a nonzero $x$ in $\operatorname{Ker} \Gamma_{e}$. Then $\varphi_{\sigma_{e}}\left(x^{\sigma_{e}} \otimes x^{\sigma_{e}{ }^{0}}\right)=0$ by explicit calculation.

Corollary. $k$ field. If $C$ is modularly semi-rigid, all $k$ separable subalgebras $B$ of $C$ are central.

Proof. Since $C$ is modularly semi-rigid, in particular ( $)^{\sigma_{e}}$ is dense for all Waterhouse two-cocycles $\sigma_{e}$. By Remark 5.2a we thus have $\varphi_{\sigma_{e}}$ injective for all $\sigma_{e}$. Hence all $k$-separable subalgebras of $C$ are central by the lemma.

We have thus shown

THEOREM 5.9. $k$ field. Let $C$ be a k-algebra with $J(C)$ nilpotent and $C / J(C)$ locally finite. The following are equivalent:

(a) $C$ is modularly rigid.

(b) $C$ is modularly semi-rigid.

(c) All k-separable subalgebras of $C$ are central.

(d) $\varphi_{o}$ is an isomorphism for all C-two-cocycles $\sigma$.

6. Categorical rigidity. In this section we take a "functorial" approach to multiplication alteration by two-cocycles. As in $\S 4$ we let $A(C)$ denote the category of $k$-algebras over $C$. Recall that given a $C$-two-cocycle $\sigma$ and an object $C \stackrel{f}{\rightarrow} D$ of $A(C), f(\sigma)$ is a $D$-two-cocycle and $C^{\sigma} \stackrel{f^{o}}{\rightarrow} D^{f(\sigma)}$ is an object of $A\left(C^{\sigma}\right)$ with $f^{o}\left(x^{o}\right)=f(x)^{f(\sigma)}$ 
for $x$ in $C$. This map describes a faithful functor from $A(C)$ to $A\left(C^{\sigma}\right)$ which we denoted $F^{\sigma}$.

Definition 6.1 (Sweedler). Let $C$ be a $k$-algebra. We say that $C$ is categorically rigid (categorically semi-rigid) if $F^{\sigma}$ is an equivalence (dense) for all $C$-two-cocycles $\sigma$.

Note that categorical rigidity implies categorical semi-rigidity. We will later show that for certain types of algebras over a field $k$, e.g., finite dimensional ones, categorical rigidity is equivalent to categorical semi-rigidity.

Suppose $\sigma, \tau$ are $C$-two-cocycles with $\sigma \sim^{\delta} \tau, \delta=\sum_{i} u_{i} \otimes v_{i}$. Then the map $R^{\delta}: C^{\sigma} \rightarrow C^{\tau}$ given by $x^{\sigma} \mapsto\left(\sum_{i} u_{i} x v_{i}\right)^{\tau}$ induces a functor $A\left(C^{\tau}\right) \stackrel{\mathscr{R}^{\delta}}{\longrightarrow} A\left(C^{o}\right)$ by "composition." For $C \stackrel{f}{\rightarrow} D$ in $A(C)$, define

$$
T_{C} \stackrel{f}{\rightarrow}: F^{o}(C \stackrel{f}{\longrightarrow} D) \longrightarrow \mathscr{R}^{\delta} F^{\tau}(C \stackrel{f}{\longrightarrow} D)
$$

to be

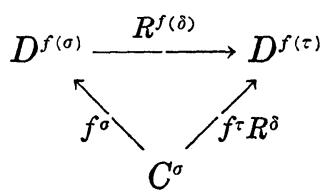

in $A\left(C^{\sigma}\right) . \quad T$ describes a natural transformation $F^{o}$ to $\mathscr{R}^{\delta} F^{\tau}$. If $\delta$ is vertible the reader may check that $T$ is a natural equivalence. Since $\mathscr{R}^{\delta}$ is an equivalence when $\delta$ is vertible we have

LeMma 6.2. Let $\sigma, \tau$ be C-two-cocycles with $\sigma \sim^{\delta} \tau, \delta$ vertible. Then $F^{\sigma}$ is dense (resp. full) iff $F^{\tau}$ is dense (resp. full).

We now direct our attention to the structure of algebras over a field $k$ which are categorically rigid.

LEMMA 6.3. Let $C$ be an algebra over the commutative ring $k$, Suppose $\sigma=\sum_{i} a_{i} \otimes b_{i} \otimes c_{i}$ is a C-two-cocycle with $e_{\sigma}=1$. Let

$$
w_{o}=\sum_{i_{1}, i_{2}, i_{3}, i_{4}}\left(a_{i_{1}} a_{i_{2}} a_{i_{3}} a_{i_{4}}\right)^{0} \otimes b_{i_{4}} \otimes\left(c_{i_{4}} b_{i_{3}}\right)^{0} \otimes c_{i_{3}} b_{i_{2}} \otimes\left(c_{i_{2}} b_{i_{1}}\right)^{0} \otimes c_{i_{1}}
$$

in $\left(C^{0} \otimes C\right)^{\otimes 3}$. Then if $w_{o}$ is invertible there is a $C^{\sigma}$-two-cocycle $\tau$ with $F^{\tau} \circ F^{o}=F^{1 \otimes 1 \otimes 1}$, which is the identity functor on $A(C)$.

Proof. There is an element

$$
w_{o}^{-1}=\sum_{j} u_{j}^{0} \otimes v_{j} \otimes w_{j}^{0} \otimes x_{j} \otimes y_{j}^{0} \otimes z_{j}
$$


in $\left(C^{0} \otimes C\right)^{\otimes 3}$ with $w_{o}^{-1} w_{o}=1$. This implies

$$
\begin{aligned}
& \sum a_{i_{1}} a_{i_{2}} a_{i_{3}} a_{i_{4}} u_{j} v_{j} b_{i_{4}} \otimes c_{i_{4}} b_{i_{3}} w_{j} x_{j} c_{i_{3}} b_{i_{2}} \otimes c_{i_{2}} b_{i_{1}} y_{j} z_{j} c_{i_{1}} \\
& =1 \otimes 1 \otimes 1 .
\end{aligned}
$$

Thus we will be done if we show that

$$
\tau=\sum_{j}\left(u_{j} v_{j}\right)^{\sigma} \otimes\left(y_{j} x_{j}\right)^{\sigma} \otimes\left(y_{j} z_{j}\right)^{\sigma} \equiv \sum_{j} d_{j}^{\sigma} \otimes e_{j}^{\sigma} \otimes f_{j}^{\sigma}
$$

is a $C^{o}$-two-cocycle with $e_{\tau}=1$.

Let $C\{X, Y, Z\}$ be the free algebra on noncommuting indeterminants $X, Y, Z$ as in Lemma 4.2. From the last paragraph we have $\left(C\{X, Y, Z\}^{\sigma}\right)^{\tau} \stackrel{\rightarrow}{\rightarrow} C\{X, Y, Z\}$ as $k$-algebras via $\left(x^{\sigma}\right)^{\tau} \mapsto x$ for $x$ in $C\{X$, $Y, Z\}$. In particular, $\left(C\{X, Y, Z\}^{\circ}\right)^{\tau}$ is an associative algebra with unit element 1. This two-cocycle unitary property for $\tau$ is then a consequence of $\left(1^{\sigma}\right)^{\tau} *\left(X^{\sigma}\right)^{\tau}=\left(X^{\sigma}\right)^{\tau}=\left(X^{\sigma}\right)^{\tau} *\left(1^{\sigma}\right)^{\tau}$. Since $\left(C\{X, Y, Z\}^{\sigma}\right)^{\tau}$ is associative we have

$$
\begin{aligned}
& \left.\left.\sum\left[\mathrm{I}\left[\mathrm{[}\left[\left(d_{i}^{\sigma} * d_{j}^{\sigma}\right) * X^{\sigma}\right] * e_{j}^{\sigma}\right] * Y^{\sigma}\right] *\left(f_{j}^{\sigma} * e_{i}^{\sigma}\right)\right] * Z^{\sigma}\right] * f_{i}^{\sigma} \\
& \quad=\sum\left[\left[\left[\left[\left[d_{\imath}^{\sigma} * X^{\sigma}\right] *\left(e_{i}^{\sigma} * d_{j}^{\sigma}\right)\right] * Y^{\sigma}\right] * e_{j}^{\sigma}\right] * Z^{\sigma}\right] *\left(f_{j}^{\sigma} * f_{i}^{\sigma}\right) .
\end{aligned}
$$

The two-cocycle associativity relation for $\tau$ follows from this.

We have left the tedious verifications to the reader since they are straightforward applications of the two-cocycle relations for $\sigma$ and the invertibility of $w_{\sigma}$.

Corollary. Let $C$ be commutative k-algebra and $\sigma$ be an Amitsur two-cocycle (i.e., an invertible $C$-two-cocycle). Then $F^{\sigma}$ is an equivalence.

Proof. Since $C$ is commutative, $e_{\sigma}$ is invertible and hence we may assume $e_{\sigma}=1$ by Lemma 6.2. $w_{\sigma}$ is clearly invertible so by Lemma 6.3 there is a $C^{\sigma}$-two-cocycle $\tau$ with $F^{\tau} \circ F^{\sigma}=F^{1 \otimes 1 \otimes 1}$. Thus $F^{\tau}$ is dense and $F^{\sigma}$ is full. It is easy to see that $\tau$ is an invertible $C^{\sigma}$-two-cocycle by its construction and another application of Lemma 6.3 proves that $F^{\tau}$ is full. Hence $F^{\tau}$ is an equivalence, which implies that $F^{\circ}$ is dense.

THeOREM 6.4. Let $C$ be an algebra over a field $k$ with $J(C)$ nilpotent and $C / J(C)$ locally finite. Then $C$ has no k-separable subalgebras (except $k$ ) $\Leftrightarrow$ all $C$-two-cocycles are invertible.

Proof. $(\Leftrightarrow)$ If $C$ had a nontrivial $k$-separable subalgebra $B$, any Waterhouse $B$-two-cocycle $\sigma_{e}$ would be a nontrivial idempotent element of $C \otimes C \otimes C$ and hence would not be invertible. 
$\Leftrightarrow$ As in the proof of Lemma 4.8, let $\sigma=\sum_{i=1}^{n} a_{i} \otimes b_{i} \otimes c_{i}$ be a $C$-two-cocycle and let $D$ be the subalgebra of $C$ generated by $\left\{a_{i}, b_{i}, c_{i}, e_{\sigma}\right\}_{i=1}^{n} \cup J(C)$. If $\bar{S}$ is any $k$-separable subalgebra of $D / J(D)$, we may lift $\bar{S}$ isomorphically to a $k$-separable subalgebra $S \leqq D \subseteq C$ by the Wedderburn Principal Theorem. By hypothesis, $S=k$ so $\bar{S}=k$. It follows from Wedderburn-Artin structure theory that $D / J(D)$ is a purely inseparable field extension of $k$. Since $J(D)$ is nilpotent $\sigma$ is invertible iff $\bar{\sigma}=p(\sigma)$ is invertible, where $p: D \rightarrow D / J(D)$ is the natural map. But $\bar{\sigma}$ is a $D / J(D)$-two-cocycle and hence invertible $[7,2.15]$.

Note that $D / J(D)$ commutative implies that $e_{\sigma}$ is invertible and $\sigma$ is equivalent to a $C$-two-cocycle $\tau$ with $e_{\tau}=1$ (cf. $\S 2$ ).

THEOREM 6.5. Let $C$ be an algebra over a field $k$ with $J(C)$ nilpotent and $C / J(C)$ locally finite. If all C-two-cocycles are invertible, $C$ is categorically rigid.

Proof. Let $\sigma$ be a $C$-two-cocycle. By the remark at the end of Theorem 6.4 and Lemma 6.2 we may assume that $e_{\sigma}=1$. Let $D$ be as in Theorem 6.4 and consider the element $w_{\sigma}$ in $\left(D^{\circ} \otimes D\right)^{\otimes 3}$ as in Lemma 6.3. Since $J(D)$ is nilpotent, $w_{\sigma}$ is invertible iff $\bar{w}_{\sigma}=p\left(w_{\sigma}\right)$ is invertible, where $p: D \rightarrow D / J(D)$ is the natural map. Because $\sigma$ is invertible and $D / J(D)$ is commutative, $\bar{w}_{\sigma}$ is clearly invertible. Therefore by Lemma 6.3 we have a $C^{\sigma}$-two-cocycle $\tau$ with $F^{\tau} \circ F^{o}=$ $F^{1 \otimes 1 \otimes 1}$. $\quad F^{\sigma}$ is full, $F^{\tau}$ is dense, and we will be done if we show $F^{\text {r }}$ is also full, i.e. $F^{\tau}$ is an equivalence.

Let $E$ be the subalgebra of $C^{\sigma}$ generated by $\left\{d_{j}^{\sigma}, e_{j}^{\sigma}, f_{j}^{\sigma}\right\} \cup J(C)^{\sigma}$. Noting how $\tau=\sum_{j} d_{j}^{\sigma} \otimes e_{j}^{\sigma} \otimes f_{j}^{\sigma}$ arose, we have $E \cong D^{\sigma}$. Since $J(C)^{\sigma}$ is a nilpotent ideal of $E, J(C)^{\sigma} \cong J(E)$ and $E / J(C)^{o} \cong D^{\sigma} / J(C)^{\sigma}=$ $(D / J(C))^{\sigma}$. Hence $E / J(C)^{\sigma}$ is finite dimensional and it follows that $J(E)$ is nilpotent. $C^{\sigma}$ has no $k$-separable subalgebras and thus $E / J(E)$ is commutative by Wedderburn-Artin theory. The invertibility of $\tau$ follows easily from the proof of Lemma 6.3 and it follows that $w_{\tau}$ is invertible in $\left(E^{0} \otimes E\right)^{\otimes 3}$. Thus $F^{\tau}$ is full by Lemma 6.3.

Now we use Waterhouse two-cocycles to prove the converse of the above theorem.

Lemma 6.6. Let $k$ be a field, $C$ be a k-algebra, and $B$ a k-separable subalgebra with separability idempotent $e$ and associated Waterhouse two-cocycle $\sigma_{e}$. Then, if $F^{\sigma_{e}}$ is dense, $B=k$.

Proof. Let $E=\operatorname{End}_{k}\left(C^{\sigma_{e}}\right)$ and $C^{\sigma_{e}} \hookrightarrow E$ be given by $x^{\sigma_{e}} \mapsto$ (left multiplication by $x^{\sigma_{e}}$ ). There is an object $C \stackrel{f}{\rightarrow} D$ in $A(C)$ with 


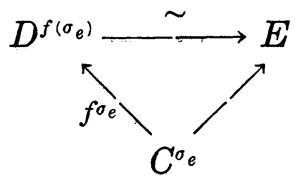

commutative. In particular, $f$ is injective.

$\bar{B}=f(B)$ is a $k$-separable subalgebra of $D$ with separability idempotent $\bar{e}=f(e)$ and associated Waterhouse two-cocycle $\bar{\sigma}=f\left(\sigma_{e}\right)$. Thus we have $D^{\bar{c}}=Z_{\bar{D}}(\bar{B})^{\bar{\sigma}} \oplus\left(\operatorname{Ker} \Gamma_{\bar{e}}\right)^{\bar{\sigma}}$ with $\left(\operatorname{Ker} \Gamma_{\bar{e}}\right)^{\bar{\sigma}}$ an ideal of square zero. Since $J\left(D^{\bar{\sigma}}\right)=\{0\}^{\bar{\sigma}}$ and $Z\left(D^{\bar{\sigma}}\right)=k$, we have $f(B)=\bar{B} \subseteq k$. Because $f$ is an injective $k$-algebra map we have $B=k$.

Combining the above results we have

THEOREM 6.7. Let $C$ be an algebra over the field $k$ with $J(C)$ nilpotent.

Consider the following statements:

(1) $\sigma \sim \delta_{\sigma} 1 \otimes 1 \otimes 1$ for some vertible $\delta_{o}$ for all $C$-two-cocycles $\sigma$.

(2) $C$ is categorically rigid.

(3) $C$ is categorically semi-rigid.

(4) $C$ has no $k$-separable subalgebras (except $k$ ).

(5) All $C$-two-cocycles are invertible. Then

(a) $(1) \Rightarrow(2) \Rightarrow(3) \Rightarrow(4) \Longleftarrow(5)$.

(b) If $C / J(C)$ is locally finite, (2)-(5) are equivalent.

Proof. (a) (1) $\Rightarrow$ (2) follows from Lemma 6.2. (2) $\Rightarrow(3)$ is trivial and $(3) \Rightarrow(4)$ holds by Lemma 6.6. For $(5) \Rightarrow(4)$, see Theorem 6.4, proof of $(\Longleftarrow)$.

(b) $(2) \Rightarrow(3) \Rightarrow(4) \Longleftarrow(5)$ by part (a). (4) $\Rightarrow(5)$ and (5) $\Rightarrow(2)$ follow from Theorem 6.4 and Theorem 6.5, respectively.

\section{REFERENCES}

1. A. A. Albert, Structure of Algebras, Amer. Math. Soc. Colloq. Pub., 24, New York, 1939.

2. S. A. Amitsur, Simple algebras and cohomology groups of arbitrary fields, Trans. Amer. Math. Soc., 97 (1959), 73-112.

3. M. Auslander and 0. Goldman, The Brauer group of a commutative ring, Trans. Amer. Math. Soc., 97 (1960), 367-409.

4. C. Curtis and I. Reiner, Representation Theory of Finite Groups and Associative Algebras, Interscience, 1962.

5. I. Herstein, Noncommutative rings, Math. Assoc. of Amer. Carus Monograph, No. $15,1968$.

6. A. Rosenberg and D. Zelinsky, Cohomology of infinite algebras, Trans. Amer. Math. Soc., 82 (1956), 85-98. 
7. M. E. Sweedler, Multiplication alteration by two-cocycles, Illinois J. Math., 15, No. 2 (1971), 302-323.

8. —, Purely inseparable algebras, J. Algebra, 35 (1975), 342-355.

Received November 19, 1976. The material in this paper forms part of a $\mathrm{Ph} . \mathrm{D}$. thesis submitted to Cornell University.

CORNELL UNIVERSITY

ITHACA, NY 14853 



\section{PACIFIC JOURNAL OF MATHEMATICS}

\section{EDITORS}

RICHARD ARENS (Managing Editor)

University of California

Los Angeles, California 90024

C. W. CURTIS

University of Oregon

Eugene, OR 97403

C. C. MOORE

University of California

Berkeley, CA 94720

\section{J. DugundJI}

Department of Mathematics University of Southern Californıa Los Angeles, California 90007

R. FinN AND J. Milgram Stanford University Stanford, California 94305

\section{ASSOCIATE EDITORS}

E. F. BECKENBACH

B. H. NEUMANN

F. WOLF

K. YOSHIDA

\section{SUPPORTING INSTITUTIONS}

UNIVERSITY OF BRITISH COLUMBIA CALIFORNIA INSTITUTE OF TECHNOLOGY UNIVERSITY OF CALIFORNIA MONTANA STATE UNIVERSITY UNIVERSITY OF NEVADA, RENO NEW MEXICO STATE UNIVERSITY OREGON STATE UNIVERSITY UNIVERSITY OF OREGON OSAKA UNIVERSITY
UNIVERSITY OF SOUTHERN CALIFORNIA STANFORD UNIVERSITY UNIVERSITY OF TOKYO UNIVERSITY OF UTAH WASHINGTON STATE UNIVERSITY UNIVERSITY OF WASHINGTON AMERICAN MATHEMATICAL SOCIETY NAVAL WEAPONS CENTER 


\section{Pacific Journal of Mathematics}

\section{Vol. 71, No. $1 \quad$ November, 1977}

Charalambos D. Aliprantis and Owen Sidney Burkinshaw, On universally complete Riesz spaces ............................. 1

Stephen Richard Bernfeld and Jagdish Chandra, Minimal and maximal solutions of nonlinear boundary value problems .................

John H. E. Cohn, The length of the period of the simple continued fraction of

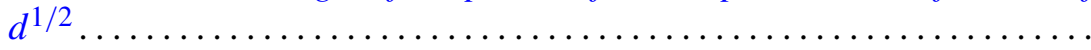

Earl Vern Dudley, Sidon sets associated with a closed subset of a compact abelian group .................................... 33

Larry Finkelstein, Finite groups with a standard component of type $J_{4} \ldots \ldots$

Louise Hay, Alfred Berry Manaster and Joseph Goeffrey Rosenstein, Concerning partial recursive similarity transformations of linearly ordered sets .......................................

Richard Michael Kane, On loop spaces without $p$ torsion. II ............

William A. Kirk and Rainald Schoneberg, Some results on

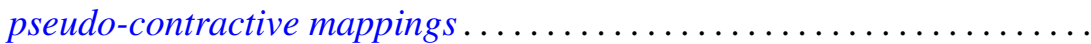

Philip A. Leonard and Kenneth S. Williams, The quadratic and quartic character of certain quadratic units. I. .

Lawrence Carlton Moore, A comparison of the relative uniform topology and the norm topology in a normed Riesz space .................

Mario Petrich, Maximal submonoids of the translational hull 119

Mark Bernard Ramras, Constructing new R-sequences . . .

Dave Riffelmacher, Multiplication alteration and related rigidity properties

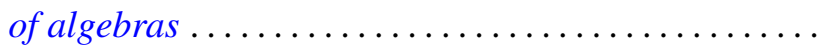

Jan Rosiński and Wojbor Woyczynski, Weakly orthogonally additive functionals, white noise integrals and linear Gaussian stochastic processes.

Ryōtarō Satō, Invariant measures for ergodic semigroups of operators

Peter John Slater and William Yslas Vélez, Permutations of the positive integers with restrictions on the sequence of differences...

Edith Twining Stevenson, Integral representations of algebraic cohomology classes on hypersurfaces ........................

Laif Swanson, Generators of factors of Bernoulli shifts . 